\title{
Discussion and Analysis on Problems of Tailored Taxi Operation
}

\author{
Fang Zhang \\ School of Humanity and Law and Economics \\ Wuhan University of Science and Technology \\ Wuhan, China 430065
}

\begin{abstract}
Didi Tailored Taxi is a new business brand launched in August 19, 2014 by the company that Didi taxihailing apps belong for high and mid grade groups to rent. Since the appearance of this "tailored taxi" service, it has been acknowledged by more and more people because it greatly alleviates the problem of difficulty in taking a taxi in big cities. However, even though the tailored taxi has the outwear of "Internet Company Certification" and makes travel of citizens convenient, transportation management departments in cities think it still needs discussion on whether the tailored taxi service is legal or not even though it "puts on the vest".
\end{abstract}

Keywords-tailored taxi; taxi-hailing apps; tailored taxi operation; tailored taxi supervision

\section{INTRODUCTION}

\section{A. Operation Pattern Classification}

According to the source of tailored taxi, there are three tailored taxi service patterns:

- use cars of the company to carry out tailored taxi service;

- use cars of the leasing company to provide service, namely the taxi-hailing apps provide information platform, the automobile rental corporation provides cars and labor service company sends drivers to finish the tailored taxi service;

- personal cars provide service. Because the number of rental car is limited, some private cars are attached to leasing company to connect with the platform of taxihailing apps.

\section{B. Legal Relation of Tailored Taxi Operation}

Tailored taxi service involves relation of multiple parties. The first party is the passenger. The second party is automobile rental corporation. The third party is the labor service company. The fourth party is taxi-hailing apps company. The relation between passenger and automobile rental corporation is the automobile leasing relation. Labor service companies send drivers. Labor service companies and automobile rental corporation form labor dispatching relation. Taxi-hailing apps provide information communication service for passengers,

About the author: Zhang Fang, master degree candidate of School of Humanity and Law and Economics, Wuhan University of Science and Technology, research direction: criminal procedure law. automobile rental companies and labor service companies. Taxi-hailing apps, passengers, automobile rental corporation and labor service companies form the relation of information service. In this way, taxi-hailing apps company is only an information platform, responsible for providing related information of rental car and drivers of the labor service companies to integrate passenger service and provide paid service for the society.

\section{Characteristics of Tailored Taxi Service}

"Tailored taxi" service mainly has the following characteristics: firstly, passengers do not have to wait for the car on the road so that it saves time; secondly, passengers can realize planned travel and order the car in advance at any time; thirdly, the "tailored taxi" fare is open and transparent. Passengers can calculate the "tailored taxi" fare in advance through the estimation system. Different levels of "tailored taxi" service are provided for passengers who can cancel the service at any time; fourthly, the "tailored taxi" service has complete evaluation system, responsibility investigation and rewards and punishments system, stimulates the enthusiasm of passengers to participate in the evaluation and marking of "tailored taxi" service through giving lottery ticket and accumulated points for them, and establishes mechanism of assessment and lowliest place elimination of "tailored taxi" service; fifthly, the types of "tailored taxi" are rich, including cars and business purpose vehicles with different grades for passengers to choose; sixthly, enhance the propaganda through marketable operation and coupon rewards to make more and more passengers willing to use "tailored taxi". To ensure the safety of passengers, related companies also provide insurance service particularly.

\section{DISCUSSION ON PROBLEMS IN DEFINITION OF TAILORED TAXI}

\section{A. Legal Qualitative Determination Is Unclear}

On March 12, Yang Chuantang, the Minister of Transport says during the two sessions, "Private cars are never allowed to serve as tailored taxi." He also declares the related instruction opinions on taxi industry will be introduced this year. Undoubtedly, rapid development of tailored taxi market will inevitably cause reform. But as an emerging industry, tailored taxi industry should seriously think about how to balance the 
relation between market operation of tailored taxi and national macro control.

Operators of taxi-hailing apps think they only provide a platform for passengers to reserve taxies, and "tailored taxi" service is the pattern that passengers, leasing company and labor service companies achieve the common purpose through the platform. It combines rental service and designated driver service and mainly pays attention to the demands of passengers. However, on specific implementation mode, the industry attribute of "tailored taxi" service is unclear. If define it as automobile leasing, it violates the rule that drivers cannot be provided; if define it as taxi, it does not conform to the provision of the state on market access and operation of taxi industry.

\section{B. Difference between Tailored Taxi and Unlicensed Cab}

At present, only Qiqihar City of Heilongjiang Province clearly regards "tailored taxi" as unlicensed cab and issues standard documents of local government - Announcement on Strictly Prohibiting Automobile Rental Corporation and Private Car Owners to Undertake Illegal Transport Business. The first three articles expressly stipulate, "Private cars are strictly prohibited to use internet and mobile software to undertake operating activities of reserving taxi illegally. Once they are verified in providing the service illegally, they will be punished according to relevant provisions of Road Transportation Regulation of People's Republic of China. Those whose actions constitute crimes shall be investigated for their criminal responsibility according to law. Automobile rental corporations are forbidden to use private cars or other non rental cars to provide service illegally by surpassing the scope of registration and provide convenience for people who undertake taxi operation illegally in the name of automobile leasing. Relevant websites and apps operators are forbidden to provide or install taxi-hailing apps for vehicles and people without business qualification to engage in illegal operating activities."

The lawyer Chen Zhihui from Beijing Tiantong Law Firm thinks the law-enforcing department involving in "the first national case of tailored taxi" settles the case by regarding "tailored taxi" as unlicensed cab. In reality, tailored taxi is not equal to unlicensed cab. They have obvious differences:

Firstly, from the perspective of business qualification of taxi, both unlicensed cab and "tailored taxi" are cars without license of taxi, but the difference is that unlicensed cabs do not have any qualification; while the "tailored taxi" involved in the "first national case of tailored taxi" firstly finds a car rental firm to "attach to" and then provides service for passengers in the way of labor dispatching through tailored taxi company and using taxi-hailing apps.

Secondly, from the perspective of profit making, both "tailored taxi" and unlicensed cab provide paid service, but unlicensed cabs may take advantage of precarious situation of passengers and often ask for fare higher than that of the licensed taxi; while the "tailored taxi" service involved in the "first national case of tailored taxi" will allow passengers to choose freely according to vehicle model and condition and give preferential benefit for passengers. The fare finally paid by the passengers will be lower than licensed taxi. Moreover, user experience of tailored taxi is better than that of the licensed taxi in cleanliness of the vehicle, hardware facilities and fare.

Finally, from the perspective of safety, taxi companies are totally under the supervision of government. It has strict access system for taxi companies and taxi drivers, which is safer; while unlicensed cabs are unsafe in vehicle condition and supervision. Different from unlicensed cabs, "tailored taxi" has safe vehicle condition. Detailed information of vehicles and drivers can be monitored because they are registered in corresponding operating companies.

\section{Analysis on The Possibility Trend of Qualitative Determination of Tailored Taxi}

Since the end of October of last year, cities such as Shenyang, Nanjing, Shanghai, Jinan, Chengdu and Qingdao declared successively that tailored taxi service belongs to "illegal operation", which make "tailored taxi" come to a deadlock. In May 7, Passenger Taxi Administrative Office of Wuhan City requires Didi Tailored Taxi to clear vehicles and drivers that engage in illegal operation on taxi-hailing apps platform. In May 30, the vehicle-mounted GPS on 8,543 taxies in Jinan receives notification sent from 37 taxi companies jointly to ask all drivers to unload taxi-hailing apps in mobile phone. In June 2, Beijing Transport Administration Commission makes appointments and discusses with responsible person of the "Didi Tailored Taxi" platform and clearly proposes the company violates the provisions of existing laws and regulations that the "Didi Tailored Taxi" business uses private cars and drivers of rental cars to engage in the transport service according to passengers' will and passenger service according to mileage and time. Zhao Zhanling says to journalist of this journal, at present, there isn't unified supervision standard and reform direction in national scale. Different places hold different attitudes of regulation toward online travel industries such as taxi-hailing apps and tailored taxi software. Although the legislation of different places is similar, they hold different opinions on newly sprouted things. Enforcing authorities in different places mainly suppress private cars and prevent them from being perfectly justifiable.

Why private cars are illegal? Private cars are attached to automobile rental corporation. They are illegal obviously even though they engage in leasing business. The automobile leasing industry belongs to regulatory industry. It is also illegal if they engage in passenger service without operation certificate. The number of qualified vehicles (property right belongs to the company, applying for indicator and putting on records) of automobile rental corporation is limited and cannot meet market demands. Therefore, tailored taxi platforms tacitly consent to or indulge private cars to join. To avoid risk, private cars first attach to automobile rental corporations and then receive orders through tailored taxi platforms. The driver is also the owner of car. "It is proper to regard these vehicles as unlicensed cabs and legal to fight these privates cars that serve as unlicensed cab in the name of tailored taxi." At present, there is no special definition on this service form of tailored taxi, so that tailored taxi is between the "legal" and the 
"illegal". "Cities need tailored taxi, but few cities discuss this problem seriously."

On definition of tailored taxi in law, lawyer Wang Xinliang from Shandong Xinliang Law Firm says when he is interviewed by journalist of Legal Person, the so-called "tailored taxi" is only a noun defined by tail-hailing apps companies. It is not defined by the law. Although there is no clear regulation on operation of tailored taxi, there are clear laws and regulations in our country to stipulate the operation of vehicle. Only vehicles or units with operation certificate can provide service for passengers. Therefore, vehicles are illegal if they provide service for passengers without conforming to related provisions of law.

Delightfully, when "tailored taxi" is besieged on all sides, things turn the corner. As early as January 8, 2015, the Ministry of Transport clearly declared, "Tailored taxi" service plays a positive role in meeting the demands of transportation market on high quality, diversification and difference. "Tailored taxi" software companies should follow rules of the transportation market and undertake corresponding responsibilities. Private cars are forbidden to involve in the operation of platform. In the critical moment, the Ministry of Transport makes authoritative declaration to "encourage innovation but forbid private cars to participate in operation of "tailored taxi", which is a good signal. Meanwhile, it is also impossible to turn "unlicensed cab" into a legal one. In the future, the "tailored taxi" service may be standardized gradually.

According to media report, in the afternoon of June 3, the Ministry of Transport holds a seminar to discuss opinions on deep reform of taxi, involving the upcoming management method of car rental. The existing taxi may be divided into cruise taxi and network appointment taxi. "Tailored taxi" is brought into the category of network appointment taxi and the price depends on vehicle model. The access threshold of tailored taxi will be improved greatly. The basic requirements include the vehicles have complete formalities and operation certificate. The drivers shall get qualification of post, but they are prohibited to attract passengers through "wandering". Private cars are not allowed to join in.

Until October 8, the internet tailored taxi suspected as "unregistered household" all the time finally has "Identity Card". Shanghai Municipal Transportation Commission declares to issue No. "5952" "Shanghai Taxi Business Qualification Certification" to Didi and Kuaidi tailored taxi platforms that can carry out business of "network appointment taxi platform". This is the first "Identity Card" of internet tailored taxi platform in China. It means Didi tailored taxi and No. 1 tailored taxi have become the first batch of internet tailored taxi acknowledged by local regulators.

No matter the government is initiative or forced by the situation to introduce policies and administrative provisions, as well as standardize and reorganize taxi market including network tailored taxi, it greatly helps the benign and orderly development of tailored taxi service. From the perspective of the current situation, tailored taxi can only provide service according to the existing rules. We believe an increasing number of positive changes will happen and the legalization of tailored taxi also can be expected soon.

\section{PROBLEMS IN TAILORED TAXI SUPERVISION}

\section{A. Legal Liability Is Unclear}

In "tailored taxi" service, passengers have the right as principal to enjoy rental service and designated driver service. Passengers send information to car rental platform to show the demand of going from one place to another, instead of getting rental service of vehicles. People who use the vehicle should be the passengers and the customer, but in legal relation of "tailored taxi", people who use the vehicle "are" regarded as renter of automobile rental corporation and drivers become the employees hired from labor service companies to drive for them. According to provisions of Road Traffic Safety Law, Contract Law and Law of Insurance, during automobile leasing, if traffic violation and traffic accidents occur due to the fault of renter and driver, and if leased vehicle is detained, damaged and lost due to the fault of renter and driver, the renter shall bear the responsibility in accordance with law; unless the liability for damage needs undertaking by the driver due to intentional misconduct or gross negligence, the renter shall undertake liability for damage. Therefore, as people who receive "tailored taxi" service, they become important party of civil contracts virtually and undertake many uncertain legal risks. Obviously, it makes passengers undertake too serious legal risk and legal liability. If laws and regulations related to tailored taxi do not balance rights and obligations of all parties based on equity principle, it is likely to greatly dampen the enthusiasm of passengers to use tailored taxi service, which is a hidden danger.

\section{B. Tailored Taxi Has Disadvantages}

Firstly, the operation safety management has problems. Some tailored taxies do not have complete insurance. If problems occur when carrying passengers, the settlement of claims will be difficult. Secondly, the examination of driver's qualification is unsound. According to the report, Uber has extremely low requirement for tailored taxi drivers, who are of varying quality. In June of this year, a female in Guangzhou is sexually assaulted when taking tailored taxi. Afterwards, the police investigate and find this criminal suspect has criminal record. When people choose to take tailored taxi to enjoy better environment or service than that of traditional taxi, they may face potential dangers, because different from traditional taxi, tailored taxies don't have unified mark and don't belong to any company and the drivers don't have job number. It makes passengers become suspected about tailored taxies. Besides, the safety of passengers' information should also be paid close attention to. When passengers place orders on mobile phone, it may contain dominant or recessive personal information. People also attach importance to the safety of their privacy.

\section{SugGestions ON COUNTERMEASURES OF “TAILORED TAXI" SERVICE}

In view of the current situation that "tailored taxi" service has greatly influenced taxi and auto rental market, we should 
insist on the principles of "people first, encouraging innovations, drawing on advantages and avoiding disadvantages and standardizing management", encourage and standardize model innovation of taxi and automobile lease service, completely eradicate illegal transport business that violates interests of passengers and influences fair competition order of the market, strengthen entity responsibility, legal liability and social responsibility of each party under "fourparty protocol model" and create open, fair and orderly market environment. At that time, increase transport capacity through using middle and top grade car and new energy vehicle, refer to the existing operation pattern of "tailored taxi". The government determines the indicative price and supervises strictly to meet the high and mid grade and special requirements in the market. Therefore, we suggest basing on Taxi Service Management Regulations, defining "tailored taxi" service as appointment taxi to implement industrial management and detailing market positioning, development direction, operation rules, service specification and legal responsibility and letting "tailored taxi" service develop in accordance with law.

\section{A. Formulate Instruction Opinion on Appointment Taxi}

Taxi Business Service Management Regulations of the Ministry of Transport has defined the appointment taxi. To solve problems properly, we suggest the Ministry of Transport introducing concrete instruction opinion, making legal status of appointment taxi service explicit and standardizing its development direction, price management, service specification, operation rules and legal responsibility, with price management and operation pattern as the most important. Unreasonable pricing and operation pattern will lead to new social contradictions, trigger problems that increase transport capacity in disguised form and collect "Fenzi Money" (the money borne by people to share the expenses for a joint undertaking). Mandatory indicators should be used to supervise and urge first-tier cities to give priority to develop appointment taxi.

\section{B. Standardize the Management of On-call Service Operators}

At present, on-call service operators do not accept nor abide by related rules in taxi industry. They send software to people or vehicle without leasing management qualifications and use on-call software to provide convenience for illegal transport business, disturb the order of taxi market and make life and property security of passengers threatened. Therefore, it is imperative to strengthen the management of on-call service operators. Various regions can introduce related regulations and enforce on-call service operators to put on records in traffic management departments and operate according to requirements of these departments to standardize on-call service from the source.

\section{Strengthen Industrial Management}

Firstly, establish linkage mechanism of departments. Traffic management department and information management department shall solve "tailored taxi" service problems together in accordance with the current law and management system. Information management departments supervise and urge operators of taxi-hailing apps to actively bear corresponding responsibilities and integrate in regulation system of government as soon as possible. Secondly, scientifically position the development of car rental industry and delimit automobile leasing and taxi. The author thinks automobile leasing refers to the operation activity of taxi for passenger transport that delivers the rental car to renter to use and keep and charges corresponding fees according to the contract. We suggest the Ministry of Transport introducing national unified laws and policies on car rental industry management to promote its healthy and orderly development. Automobile rental corporations shall establish leased vehicle management files, strictly forbid private cars to attach to leasing companies and dispose according to laws and regulations, cooperate with public security sector and economic and information committee to take special measures to strictly crack down illegal behaviors of rental cars and private cars to engage in illegal transport business through software.

\section{CONCLUSION}

In terms of problems facing by the development of internet tailored taxi industry, traffic management department and information management department should solve "tailored taxi" service problems together, improve the current laws and regulations and reduce "grey zone" to make the tailored taxi management have legal basis. Internet tailored taxi operation enterprises should capture market positioning, implement differentiation strategy and meet different demands of passengers, ease intertype competition and then promote the transition from "Red Sea Competition" to "Blue Sea Win-win". Moreover, they should take the initiative to assume responsibility, coordinate with supervision of government sector and strengthen the examination of qualification of tailored taxies and drivers as well as intensify safety management. Tailored taxi is a new thing and its development road will be full of twists and turns, but we should believe it has brilliant prospect. We believe tailored taxi will develop continuously under the tide of sharing economy.

\section{REFERENCES}

[1] Hu Qingbo. The First Case of Tailored Taxi: Collision of Tailored Taxi and Law [J], Law and Life, 2015,09:10-11.

[2] Zhang Hongbin. Management and Governance under the Reflection of "Tailored Taxi" [J], New Economy,2015,06:15.

[3] Xu Gaofeng. Tailored Taxi Is Suffering from Barriers of Law [J], Information Construction, 2015,02:51-52.

[4] Xu Gaofeng. Tailored Taxi Is Coming [J], Information Construction, 2015,02:45-47.

[5] $\mathrm{Xu}$ Gaofeng. Where Is the Pain Point in Supervision of Tailored Taxi [J], Information Construction, 2015,02:50-51

[6] Wang Yu. The National "First National Case of Tailored Taxi" Opens a Court Session and Will Choose a Day to Pronounce Judgment. Administrative Punishment Case of the First Tailored Taxi Service Questions Closely about Whether the "Tailored Taxi" Is Legal [J], Shanghai Enterprise, 2015,05:90-92.

[7] Huang Yuemei. Discussion on Problems of "Tailored Taxi Service" [J],Transportation Enterprise Management, 2015,05:6-7 
[8] Lei Huafang. Research on Whether the Tailored Taxi Service Is Illegal Business [J], Business, 2015,10:216

[9] Li Lijuan. Where Is the Way of "Tailored Taxi Service" [J], Legal Person, 2015,04:62-63. 